\title{
CONSISTENT MODELLING OF THE IMPACT MODEL OF MODULAR PRODUCT STRUCTURES WITH LINKING BOUNDARY CONDITIONS IN SYSML
}

\author{
Schwede, Lea-Nadine; Hanna, Michael; Wortmann, Nadine; Krause, Dieter \\ Technical University Hamburg
}

\begin{abstract}
The challenges related to product structures, which go hand in hand with megatrends such as individualization, can be met with the modularity of product structures. With the help of various modularization methods, modular product structures are created with regard to different goals. There are many references to the effects of modular product structures on life phases and economic targets in the literature. These effects were collected in previous research in a generic impact model. Since there is a lot of information about the effects, such models become very comprehensive and thereby difficult to handle. For this reason, the impact model is consistently generated using SysML. The adaptation to company scenarios is possible through the use of simulations with which, for example, company-related and product-related boundary conditions can be controlled by means of a User Interface.
\end{abstract}

Keywords: Systems Engineering (SE), Product structuring, Effects of Modularization, Consistency, Constraint modelling

\section{Contact:}

Schwede, Lea-Nadine

Technical University Hamburg

Institute of Product Development and Mechanical Engineering Design

Germany

lea.schwede@tuhh.de

Cite this article: Schwede, L.-N., Hanna, M., Wortmann, N., Krause, D. (2019) 'Consistent Modelling of the Impact Model of Modular Product Structures with Linking Boundary Conditions in SysML', in Proceedings of the 22nd International Conference on Engineering Design (ICED19), Delft, The Netherlands, 5-8 August 2019. DOI:10.1017/ dsi.2019.367 


\section{INTRODUCTION}

Globalization continues to leads to a wider range of external variety to fulfil individual customer needs. To prevent an equal growth in internal variety, the product structure of product families has to be taken into account. The reduction of internal variety can be done using modularization methods. The objective of these methods is the creation of modular product structures (Krause et al., 2014).

When modularizing a product or product family it is important to know the consequences of changing the product architecture. In particular, economic targets are important in company decision making on whether to modularize. In the literature there are a lot of independent publications that deal with the impacts of modularization and highlight specific effects (Boer, 2014; Fixson, 2006; etc).

The various considerations were combined into a comprehensive Impact Model of Modular Product Structures (Impact Model) by the Institute of Product Development and Mechanical Engineering Design (PKT), based on the literature. The Impact Model provides a holistic overview of the relationships between the properties of modular product structures, their effects on the life phases, the product development process and the associated economic effects. Boundary conditions for certain effects have also been researched (Hackl and Krause, 2017).

The developed model collates a lot of knowledge and delivers an enormous amount of data. Due to this growing amount of data it becomes difficult to use the information generated. Representation of the impact model used to date is therefore no longer practicable.

The aim of this paper is to collect and structure the data so that it can still be handled and the impact model becomes resilient to change, as it is the subject of ongoing research.

This paper shows a possible way to achieve these goals. The approach presented in this paper is the integration of the Impact Model in Cameo Systems Modeler with an adaption of the modelling language SysML. This modelling allows the consistency of the data on different levels. A major advantage is that boundary conditions can be assigned directly to effects and the model can therefore automatically be adapted to different use cases.

Section 2 presents the current state of the Impact Model, including related data. The basics of MBSE are briefly presented. The challenges of the impact model are analyzed in Section 3. Section 4 shows the preliminary work in creating a model in Cameo Systems Modeler using an adaption of SysML. The focus is on creating a data model and the derivation of implementation definitions. Section 5 shows an excerpt of the implemented Impact Model in SysML. Special attention is paid to the connection of boundary conditions and consistency. Discussion and outlook conclude the paper.

\section{THE IMPACT MODEL AND BASICS OF MBSE}

The current state of research on the Impact Model is represented. The basics of MBSE, which are relevant, are briefly presented.

\subsection{Structure of the Impact Model and related data}

The starting point for modelling in SysML is the Impact Model developed by the Institute of Product Development and Mechanical Engineering Design (PKT) to create a generic model for mapping the characteristics and effects of modular product structures and provide a system view of potential influences and their resulting links. The model shows how changes in the properties and characteristics of modular product structures affect economic factors (Hackl and Krause, 2016).

In a follow-up project, modularization methods will be adapted to the Impact Model to provide a consistent way to evaluate modularization methods. The Impact Model is divided into three main areas: properties and characteristics, life phases effects, and economic target values.

An excerpt from the Impact Model (intermediate result) is shown in Figure 1. 


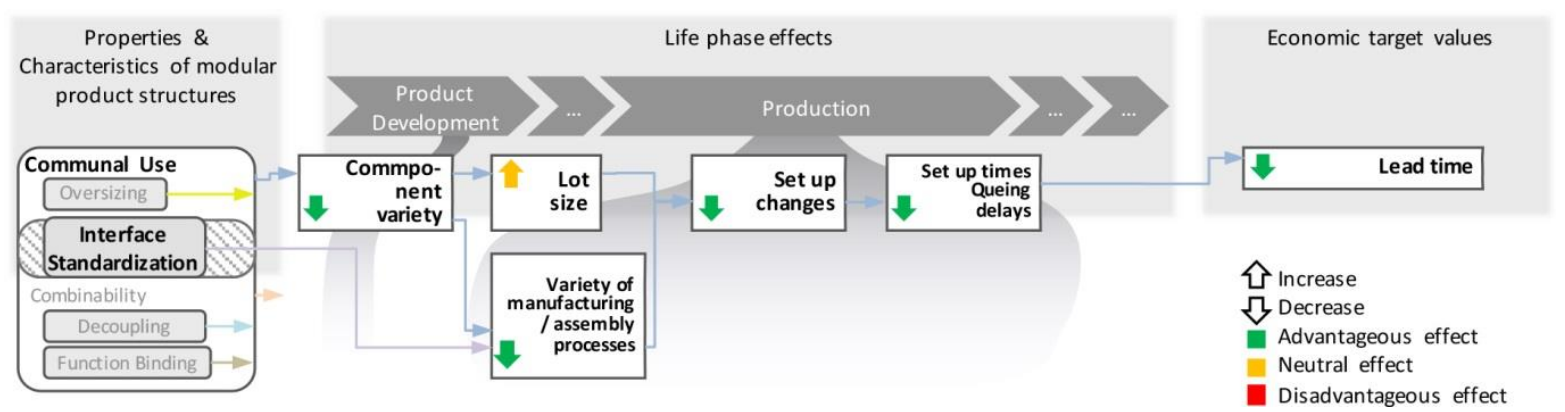

Figure 1: Shape describing excerpt of the Impact Model (intermediate result) (Hackl and Krause, 2017)

The left of the picture shows the characteristics and properties of modular product structures. Characteristics can be directly influenced by the product developer but can lead to properties that cannot be directly influenced (Weber, 2007; Salvador, 2007). An example of a modularization property is communal use, which is the most named effect driver (Hackl and Krause, 2016). A module is communal when it has multiple uses, such as in a product or a whole product family (Salvador, 2007). Interface standardization is a characteristic of modular product structures, cannot be influenced, and is required by the communal use.

Modularization characteristics and properties lead to the various effects listed in the literature, which are shown in the middle of the picture. The effects are assigned to the life phases, which are arranged chronologically (Figure 1, top, horizontally). Every effect has a small arrow that shows whether it increases or decreases (up/down), and whether it is advantageous (green), neutral (yellow) or disadvantageous (red). The effects are connected to each other and build up effect chains (Hackl and Krause, 2017).

Figure 1 is an example of an effect chain. It shows the causal relationship that the communal use of modules has a positive effect on component variety since more standard parts are available in the product family. The components therefore have higher lot sizes but fewer set-up changes in the production phase. This effect also has a connection to another effect (Variety of manufacturing/assembly processes), which is directly influenced by interface standardization. The decrease in set-up changes results in fewer delays, which means shorter lead times, which in this case is an economic target value.

The Impact Model can be used to evaluate product concepts in the course of product development. If modularization of a product family has two concepts, they can be analyzed for their characteristics and properties. Depending on which characteristic is more strongly pronounced, effects occur more or less intensively. The strength and type of effect expression also depends on boundary conditions. This creates a dependency between effects and boundary conditions. Boundary conditions in this case are the company production type, which produces the product (family) mentioned above.

\subsection{MBSE with modeling in SysML}

Originally, systems engineering was used to develop systems consistent in their behavior and architecture according to interdisciplinary systems requirements.

Model-based systems engineering (MBSE) contains, besides processes and methods, modeling with methods of systems, which include system requirements, design, analysis, verification and validation. Part of MBSE is modeling systems with all their connected information. By connecting different data in a system MBSE allows easier document management, based on a common database. Network models are created in the process (Walden et al., 2015; Holt et al., 2012; Friedenthal et al., 2009).

Various modeling languages and tools can be used to create models. One way to build models is with the software Cameo Systems Modeler (no magic) and the modeling language SysML. The modeling language SysML is based on the Unified Modeling Language (UML) (Weilkins, 2007; Holt et al., 2012).

SysML uses different kinds of diagrams to show connections between system elements. In diagrams, elements are related to each other via different connection types. There are 9 diagrams in total, which are subdivided into behavior diagrams and structure diagrams. The requirements diagram stands alone and describes the connection between requirements, sub requirements and stakeholders. 
An example of a behavior diagram is the activity diagram in which flowcharts or functional structures can be displayed. The block definition diagram (BDD) is an example of a structure diagram. It is used to show the structure of system elements and their connection to each other. The system elements in this case are blocks. A connection between blocks can be the generalization or directional composition (Object Management Group, 2017; Holt et al., 2012).

Blocks can also be related via an allocation or a dependency, which can be described more accurately using (self-defined) stereotypes. Stereotypes can be seen as categories assigned to elements. Adjustments to the SysML notation in Cameo Systems Modeler are possible. In addition to the use of stereotypes, the display of system elements, such as blocks, can also be changed and icons can be stored.

\section{ChALLENGES RELATED TO THE IMPACT MODEL}

The Impact Model generates an overall view. Since the Impact Model contains 81 effects from 39 literature sources it has an enormous presentation size. Ongoing research has shown that there are a lot of effects that have boundary conditions. Boundary conditions are not yet included directly in the model. Information additional to single interrelations might be useful to understand and work with this model. An example is information about the connection between unit costs and lot size, which is information additional to the economic effect 'Procurement costs' (Figure2).

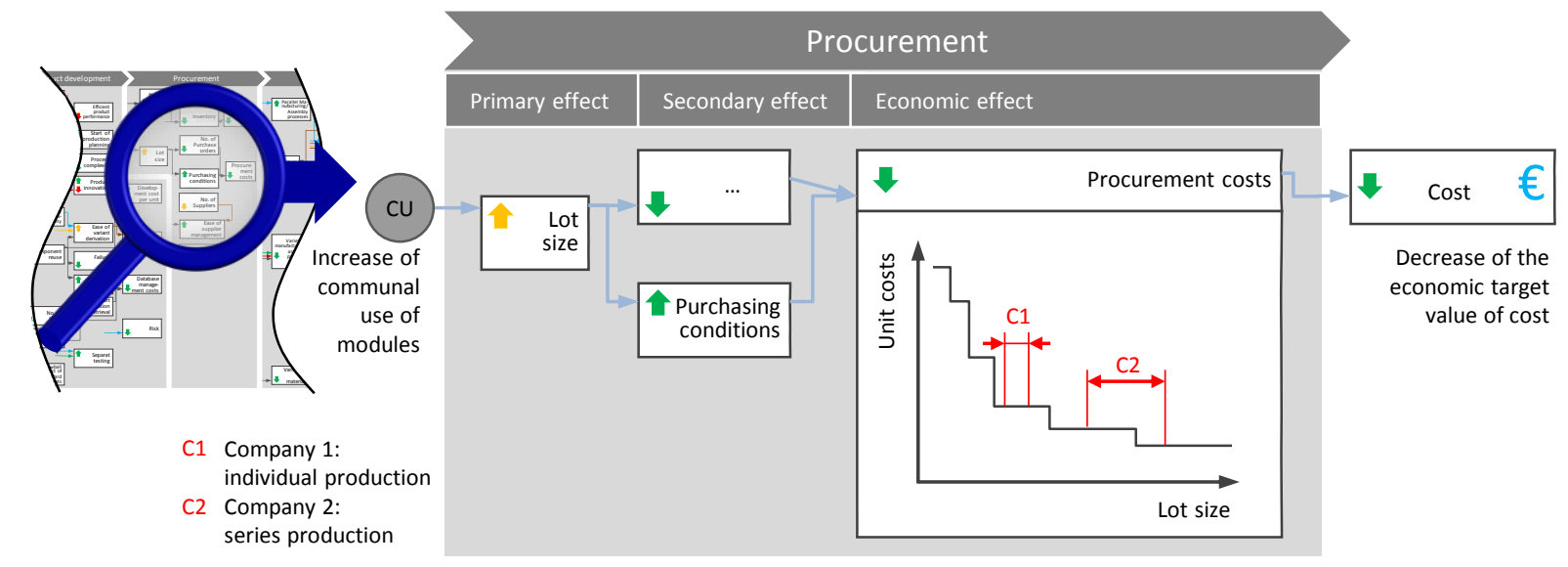

Figure 2: Excerpt of the Impact Model with a focus on an effect chain (Hackl and Krause, 2017)

This specific additional information indicates that the strength of the effect can differ depending on whether a company is using individual production $(\mathrm{C} 1)$ or series production $(\mathrm{C} 2)$. The graph shows the relation that the higher the change in lot size (due to communal use of components), the more likely that unit costs will decrease by possible discounts. Since C2 in this case can create a bigger change in the number of lot size, the effect will probably have a higher influence for $\mathrm{C} 2$ than for $\mathrm{C} 1$ (Hackl and Krause, 2017).

Up until now the Impact Model has been presented in Microsoft PowerPoint and was static and one dimensional. Additional information such as that shown in Figure 2 is not directly included in the Impact Model for the sake of clarity. Because the Impact Model is very big and the subject of ongoing research, there is more and more model data, for example, product structures and modularization methods, which complicates applicability of the model.

One-dimensional representation in Microsoft PowerPoint is no longer true to time. To make the Impact Model, with all of its additional information, more powerful, presentation with more dimensions is required.

One way to achieve this type of presentation is to generate a model that has several levels and perspectives with an adaptation of SysML in a tool like the Cameo Systems Modeler. This procedure is presented and discussed in this paper. Other possibilities, not discussed here, could be using the modeling language UML or creating models in MATLAB. 


\section{PROCEDURE FOR CREATING A MODEL WITH SYSML}

This section examines the elements needed to generate a SysML Model. A data model of the Impact Model is presented that shows which elements are connected to each other. Implementation in SysML (with adaptations) is then guided by this.

\subsection{Data model of the Impact Model}

In order to generate a data model of the Impact Model in SysML, the Impact Model has to be analyzed first. Since SysML was invented neither for product development nor for analyzing the impacts of product structures, given definitions must be adapted. It is important to know what type of connections there are between the model elements, and the properties and requirements of the model elements have to be analyzed to generate a data model on which a SysML model can be built. The data model, which can be understood as an expandable meta model, is the result of the analysis and forms the basis for modeling in SysML (Figure 3).

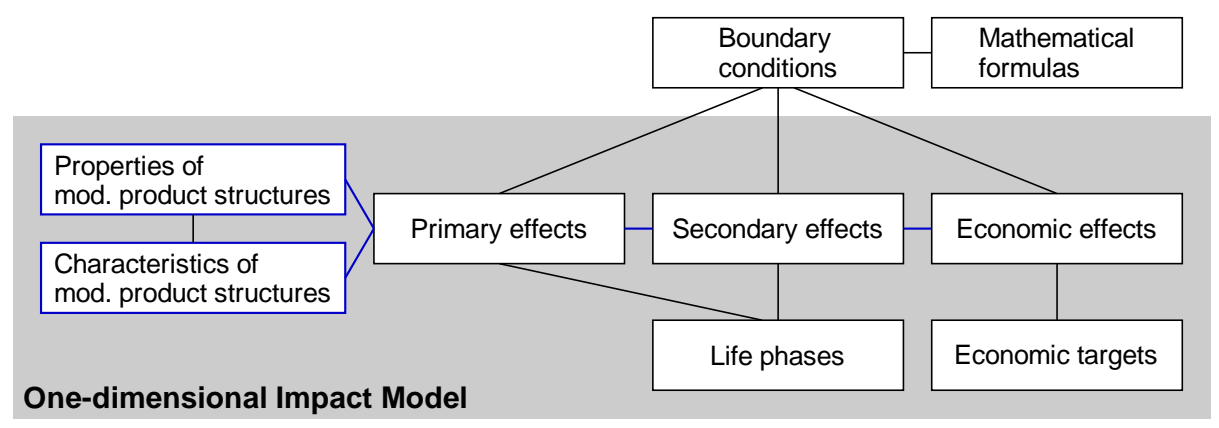

Figure 3: Data model of the Impact Model

The data model describes the relations between the system elements in a more abstractive way. The elements are connected to each other via lines. All system elements which are connected in this model have to be connectable in the following modeling in SysML. The data model in Figure 3 shows that an economic effect (such as procurement costs) has to be connectable to secondary effects, and economic targets. Connection to boundary conditions, which could also be described via mathematical formulas, has to be possible in the SysML model. The blue lines are assigned to the properties and characteristics by their coloring.

\subsection{Implementation definitions for the system elements (with adaptations of SysML)}

Based on the data model, the implementation definitions in the modeling language SysML (with adaptations) are presented in this subsection.

Because a lot of different kinds of elements have to be connected in the Impact Model (grey area in Figure 3), the block definition diagram is used for the Impact Model. The different elements are implemented as blocks since blocks are the system elements which are the most flexible in interacting with other system elements and depositing of additional attributes.

The boundary conditions are an exception. The boundary conditions are treated as requirements in the SysML context. The element requirement in SysML includes a text field to describe the requirement itself. Further additional information, which cannot be included as text in the requirements, can be easily connected to the requirements using hyperlinks to PDF documents. The modeling language SysML contains the requirements diagram to organize requirements; it is possible to refine requirements via constraints, which can be mathematical formula and therefore works perfectly for this application area.

The type of connection between the boundary connection and the effects varies. An example was given in Section 3: a boundary condition of an effect can be additional information. In this case, a requirement is connected to an effect by the link type dependency, which is modified with a stereotype in SysML.

Other boundary conditions can directly influence the expression of the effect, for example, the influence of the production kind of a certain company. In SysML, a requirement with a connected constraint is used. Predefined 'if' statements or functions constructed in MATLAB can be stored in a constraint. 


\section{THE STRUCTURE OF THE IMPACT MODEL IN SYSML}

A section of the Impact Model is shown in a block definition diagram. A deeper look is taken into the connection of the boundary conditions, which can then also be operated via a user interface. Consistency within the developed model is described in the last section.

\subsection{Block definition diagram of the Impact Model}

The SysML model in Cameo Systems Modeler has a BDD that shows the Impact Model. An excerpt of the implemented Impact Model is shown in Figure 4.

The depicted structure matches the structure shown in Figure 1 and 2 and therefore provides the same information as the static presentation of the Impact Model. Properties and characteristics of modular product structures lead to effects in life phases, which lead to economic effects. Every element in the Impact Model is defined here by the system element block. By double click it is possible to get further information about the specific element, such as the type of boundary condition and which way it is linked, or which other effect also has an influence on the effect but is not shown in the Impact Model. Since the Impact Model is based on the literature, these sources are linked to individual effects and the connections between them.

The type of link between the characteristics and properties of modular product structures (blue and purple) and the effects is dependency. Characteristics and properties were modified by defining stereotypes. This assigns categories to the dependencies. The example shown in this case is the dependency with the stereotype 'communal use' (blue, narrow arrowhead).

In the Cameo Systems Modeler coloring is possible but the color of an element is not traceable since colors don't exist in SysML.

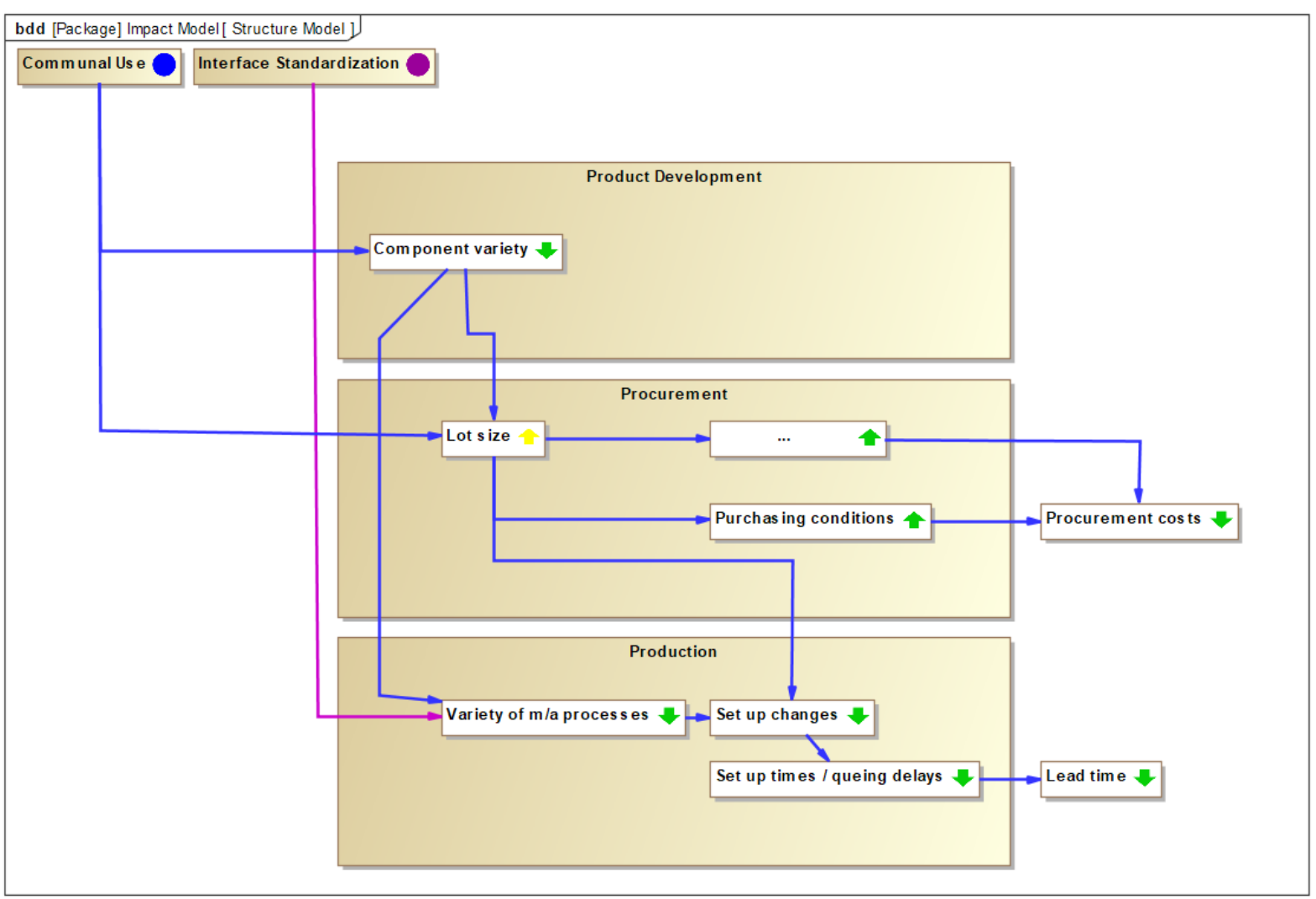

Figure 4: BDD of the Impact Model (Excerpt)

The effects are represented similarly to those in Figure 1 and 2. The colored arrow is implemented as an icon. The icon was placed as a PNG image connected to the effect and therefore stored in the model with the system element. 


\subsection{Connection of the boundary conditions}

Connection of the boundary conditions to the effects, to be able to use this information in user interfaces, plays an important role in the application of the Impact Model to specific company scenarios. One example of connecting boundary conditions to effects is shown in this subsection. The impact of the effect 'procurement costs' depends on the production type. How this boundary condition is connected is shown in Figure 5.

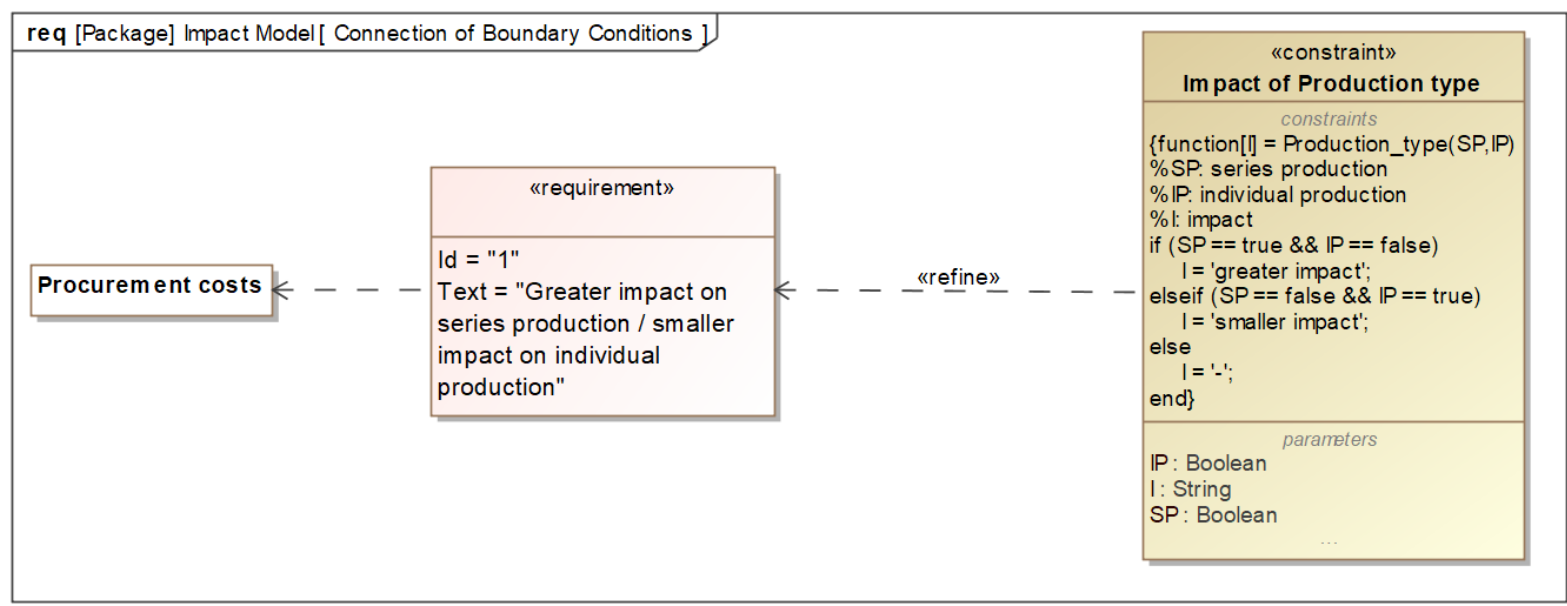

Figure 5: Connection of boundary conditions to effects

On the left is the effect 'procurement costs'. As mentioned, the SysML element for boundary conditions is the requirement. The requirement is connected to the effect with the linking type dependency. The SysML element contains an ID number and a text field. Categorization of the boundary conditions is possible by number. The boundary condition can be explained in the text field, in this case: 'Greater impact on series production / smaller impact on individual production'. In addition, this overarching correlation can be mapped in an if-statement constructed in MATLAB. The input variables are boolean values. Either series production or individual production can be chosen as true. Depending on the choice made, the output that is represented as a string value can be either 'greater impact' or 'smaller impact'. This function is stored in a constraint that is connected to the requirement by the connection type refine.

This connection is not necessarily a one-to-one assignment. A boundary condition can be connected to more than one effect. An effect does not have to be connected to just one boundary condition. If that is the case, balancing has to take place since it might be possible that different boundary conditions influence an effect in different ways.

The defined constraints and their inputs can now be controlled by user interfaces connected to simulations in Cameo Systems Modeler (Figure 6).

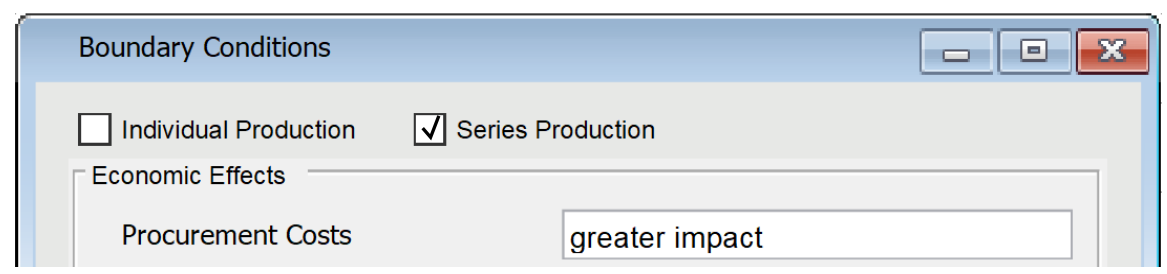

..

\begin{tabular}{|c|c|c|c|}
\hline \multicolumn{2}{|l|}{ Boundary Conditions } & $\begin{array}{ll}\square & \text { 口 }\end{array}$ & 8 \\
\hline \ Individual Production & \multicolumn{3}{|c|}{ Series Production } \\
\hline \multicolumn{4}{|l|}{ Economic Effects } \\
\hline Procurement Costs & smaller impact & & \\
\hline
\end{tabular}

Figure 6: Interface for interaction regarding boundary conditions

The inputs for the simulations are arranged on the top of the user interface. By clicking on the fields, the checkboxes can be checked. A check marks the boundary condition as true and thus applicable. The string value in the output box depends on the input checkbox. 
Simulations with user interfaces can be built on previously created SysML models. With simulations a model becomes more powerful. An additional tool is generating generally understandable knowledge, which results from the defined contexts. This can used together with the BDD that contains the Impact Model. The constraint property is deposited. Additionally, the given graph, which shows the qualitative connection between lot size and unit costs, can be deposited as additional information.

\subsection{Consistency in the context of the Impact Model}

The connections included in the BDD in Figure 4 are consistent throughout the entire model in Cameo Systems Modeler and is therefore traceable. It is possible to build up some dependency matrices that include the same information as the Impact Model. This might be useful, because the Impact Model is enormous and the connections are confusing. The dependency matrices are also very useful since the Impact Model is still a work in progress and if a new effect is required, for example, it can be easily included in the matrix by setting all the dependencies to other effects of properties and characteristics. The new effect with its connections is than automatically shown in the BDD of the Impact Model.

Generatable consistency is a major advantage of the MBSE compared to the one-dimensional Microsoft PowerPoint based presentation of the Impact Model. During the creation of a SysML model different types of consistency have to be mentioned to be able to automatically generate an overall consistent model. Hanna and Schwede used the definitions of consistency types, based on Bursac and Scherer, to describe consistency within the framework of product development with MBSE (Hanna et al., 2018; Bursac, 2016; Scherer, 2016). The different types are: consistency between models, consistency of time and consistency of vertical layers. All types are considered in the developed SysML model (Figure 7).

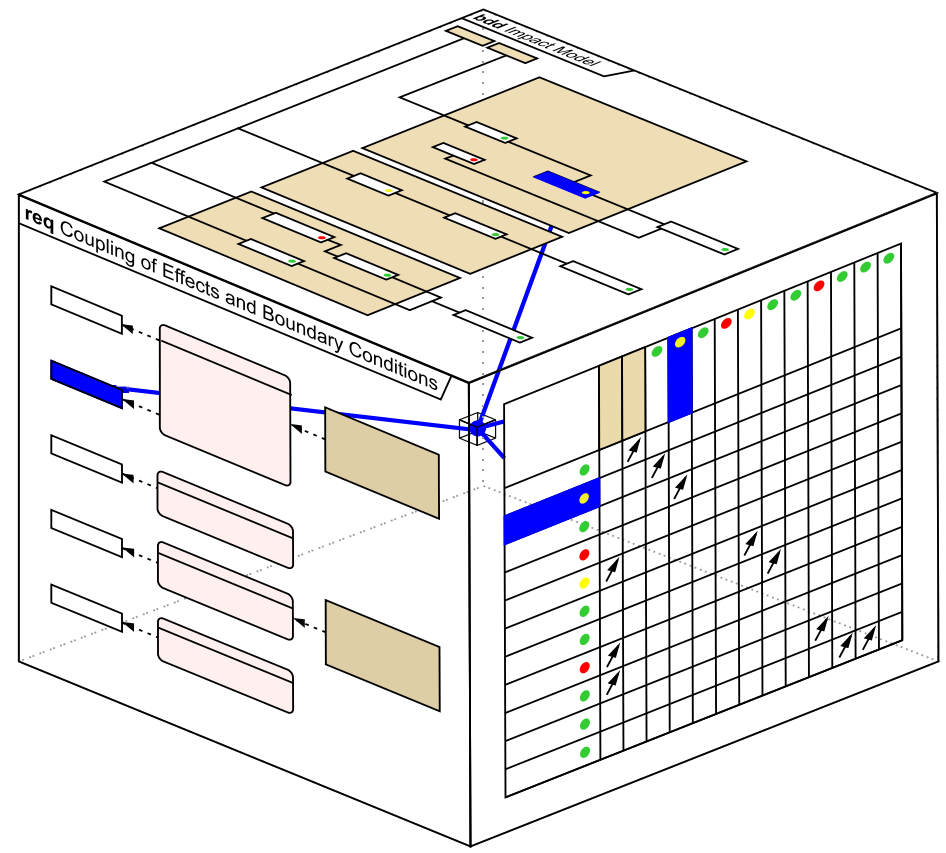

Figure 7: Visualization of the Consistency in the SysML model

Consistency between models works similarly to Hanna and Schwede's interpretation: Every element only exists once in the SysML model. Different diagrams are built upon them. If there is a change in a system element due to a change in presentation of the system element, the root element changes too. An example is shown in Figure 7. An effect (blue) represents a root element, which is used in the BDD of the Impact Model, the requirements diagram and in a dependency matrix. If the effect changes, all the visualizations in the various diagrams and matrix will change too.

In the Impact Model effects play a central role. Effects are the main elements in the presentation of the Impact Model (Figure 1). They are also part of the dependency matrix. Requirements are also connected to the effects. but occurs separately in requirements diagrams. In these diagrams, the same system element is used as in the representation of the Impact Model in the BDD.

The consistency of time is included in the model in two ways. Versioning of the Impact Model is included. Different work statuses are retrievable. This is important since research on the Impact Model 
is ongoing. The model can be used in different boundary condition situation. Cameo System Modeler stores the result of simulations automatically. If the model is used in a specific product use case of one specific company, the simulation results are storable and export of the data out of Cameo Systems Modeler to Microsoft Excel is possible.

Consistency of vertical layers is achieved using the BDD of the Impact Model as the main partial model. The diagrams that were used to define connections between the effects and boundary conditions or dependency matrices are hierarchically integrated below.

\section{DISCUSSION AND OUTLOOK}

This paper describes the transition of a one-dimensional Impact Model, represented in Microsoft PowerPoint, to a multidimensional Impact Model modeled with adapted SysML in the Cameo Systems Modeler. A SysML model delivers a lot of advantages: A lot of different data types can be connected to each other in a consistent way. If one element is changed, the change is reflected throughout the model. Since the Impact Model is the subject of ongoing research presentation of it with traceable connections is absolutely necessary to maintain an overview of these complex interrelationships. By creating a SysML model, a possibility has been developed to link new data uniformly to the Impact Model without neglecting existing information.

The Impact Model is based on a consistent data model with predefined interfaces to be able to link more data in ongoing research. Additional information can be linked to the model elements. It is possible to generate different views of the Impact Model with a different focus and validation of the Impact Model.

The SysML model combines the Impact Model as a reference guide for possible effects of modular product structures and specific impact models, derived for specific application scenarios with their unique set of boundary conditions.

With simulations and user interfaces, the model is even more useful since it turns into a tool that can be adapted to different situations. It is a way to use the huge amount of collected data and its connections in an understandable way. Easy-to-use user interfaces also reduce the inhibition threshold when dealing with SysML models.

In terms of visualization, Cameo Systems Modeler has the advantage over open source competition programs as images and colored elements can be integrated. The final one-dimensional Impact Model is transferred to a BDD so that it looks identical in the first view. The initial outlay is quite big, but later changes, such as integrating a new effect of boundary condition, can be made easily.

The set of possible boundary conditions is not yet complete and interrelationships and influences have so far only been of a qualitative nature. Ongoing research deals with deriving an extensive set. When connections between boundary conditions and effects get more mathematical, the Cameo Systems Modeler given interface to MATLAB and SIMULINK can be used.

The next steps are the connection of product structures as individual elements (product variants, modules, components) and the connection of method steps of modularization methods, which are responsible for module formation. The long-term goal is the targeted control of individual effects with the aid of method steps and thereby with the aid of selected modularization methods.

Key figures and characteristic curves will be connected in different contexts. Key figures of the properties and characteristics of modular product structures can be used to determine the degree of modularity and a lot of effects can be described by characteristic curves, which also can be included by mathematical functions. When the overall model is developed and integrated in SysML, individual impact models for application examples can be derived.

In current research different product development methods are already modeled (partly) with SysML (Bursac, 2016; Hanna and Krause, 2017; Hanna et al., 2018; etc.). Here a connection of the Impact Model is conceivable.

The Impact Model, which shows the accumulated knowledge from decades of application of modularization methods, becomes an interdisciplinary usable tool with the help of modeling in MBSE. The Impact Model thus represents a powerful tool for method applicators who want to demonstrate and control the effects of the method application. 


\section{REFERENCES}

Boer, H.E.E. (2014), "Product, Organizational, and Performance Effects of Product Modularity", In: T.D. Brunoe, K. Nielsen, K.A. Joergensen and S.B. Taps, (Ed.), Proceedings of the 7th World Conference on Mass Customization, Personalization, and Co-Creation (MCPC 2014), February 4th - 7th, 2014: Twenty Years of Mass Customization - Towards New Frontiers, Lecture notes in production engineering, Springer International Publishing, Cham, Aalborg, Denmark, pp. 449-460. https://doi.org/10.1007/978-3-31904271-8 38.

Bursac, N. (2016), Model Based Systems Engineering zur Unterstützung der Baukastenentwicklung im Kontext der Frühen Phase der Produktgenerationsentwicklung, Dissertation, Karlsruher Institut für Technologie (KIT), Karlsruhe. https://doi.org/10.5445/IR/1000054484.

Fixson, S.K. (2006), “A roadmap for product architecture costing”, In: T.W. Simpson, J.R. Jiao and Z. Siddique, (Ed.), Product platform and product family design methods and applications // Product Platform and Product Family Design: Methods and Applications, Springer Science+Business Media LLC, New York [u.a.], Springer, pp. 305-334. https://doi.org/10.1007/0-387-29197-0_13.

Friedenthal, S., Steiner, R. and Moore, A. (2009), A Practical Guide to SysML - The Systems Modeling Language, Morgan Kaufmann Pub, San Francisco. https://doi.org/10.1016/C2010-0-66331-0.

Hackl, J. and Krause, D. (2016), "Effects of Modular Product Structures on Life Phases and Economic Factors", 14th International Design Conference - Design 2016, Dubrovnik, Croatia, pp. 1285-1294.

Hackl, J. and Krause, D. (2017), “Towards an Impact Model of modular Product Structures”, Proceedings of the 21st International Conference on Engineering Design, ICED17, Vol. 3, pp. 151-160.

Hanna, M. and Krause, D. (2017), "Model-based support for product family design", 20th International Dependency and Structure Modeling Conference, DSM 2017, Espoo, Finland.

Hanna, M., Schwede, L.-N. and Krause, D. (2018), "Model-Based Consistency for Design for Variety and Modularization", 20th International Dependency and Structure Modeling Conference, DSM 2018, Trieste, Italy, pp. 239-248.

Holt, J., Perry, S.A. and Brownsword, M. (2012), Model-based requirements engineering, IET professional applications of computing series, Vol. 9, Institution of Engineering and Technology, London.

Walden, D.D., Roedler, G.J., Forsberg, K., Hamelin, R.D. and Shortell, T.M. (Eds.) (2015), Systems engineering handbook: A guide for system life cycle processes and activities; INCOSE-TP-2003-002-04, 2015, 4. edition, Wiley, Hoboken.

Krause, D., Beckmann, G., Eilmus, S., Gebhardt, N., Jonas, H. and Rettberg, R. (2014), "Integrated Development of Modular Product Families: A Methods Toolkit”, In: T.W. Simpson, J. Jiao, Z. Siddique and K. Hölttä-Otto, (Ed.), Advances in Product Family and Product Platform Design: Methods \& Applications, martM, Springer New York, New York, pp. 245-270. https://doi.org/10.1007/978-1-46147937-6_10.

Object Management Group (2017), “OMG Systems Modeling Language. Version 1.5”, Available at: http://www.omgsysml.org/what-is-sysml.htm (accessed 21 March 2019).

Salvador, F. (2007), "Toward a Product System Modularity Construct. Literature Review and Reconceptualization", IEEE Transactions on Engineering Management, Vol. 54 No. 2, pp. 219-240. https://doi.org/10.1109/tem.2007.893996.

Scherer, H. (2016), Modellbasierte Methoden zur Modellierung des Zielsystems und des Funktions-GestaltZusammenhangs zur Unterstützung der Serienentwicklung von Baukästen am Beispiel von HybridTriebstrangsystemen, Dissertation, IPEK, KIT, Karlsruhe. https://doi.org/10.5445/IR/1000057850

Weber, C. (2007), "Looking at DFX and Product Maturity from the Perspective of a New Approach to Modelling Product and Product Development Processes", In: F.-L. Krause, (Ed.), The Future of Product Development: Proceedings of the 17th CIRP Design Conference, Springer-Verlag Berlin Heidelberg, Berlin, Heidelberg, pp. 85-104. https://doi.org/10.1007/978-3-540-69820-3_11.

Weilkiens, T. (2007), Systems engineering with SysMLUML: Modeling, analysis, design, The OMG press, Morgan Kaufmann OMG Press/Elsevier, Amsterdam, Boston.

\section{ACKNOWLEDGMENTS}

Thanks go to the German Research Foundation (Deutsche Forschungsgemeinschaft - DFG) for funding Project 'Entwicklung eines Wirkmodells der Eigenschaften modularer Produktstrukturen zur Bewertung methodischer Ansätze', which provided the starting point for this research. 